\title{
ACKNOWLEDGING DSMA WITH PRIORITY SCHEDULING FOR LOCAL AREA NETWORKS*
}

\author{
Maneesh Mehta and Jon W. Mark** \\ Department of Electrical Engineering \\ and \\ Computer Communications Networks Group \\ University of Waterloo \\ Waterloo, Ontario, Canada
}

\section{ABSTRACT}

A mini-slot, appended 10 the data slot in the distributed scheduling multiple access (DSMA) protocol described in [1], for the transmission of acknowledgement packets is described and analyzed. The resulting DSMA with fixed slot acknowledgement (DSMA/FSA) presents a better alternative to sending a separate acknowledgement packet for each received data packet. With priority requests, DSMA/FSA appears to be a viable media access prolocol for integrated voice and data services in a local area nelwork

\section{INTRODUCTION}

DSMA (Distributed Scheduling Multiple Access) is an asynchronous lime-division multiple access scheme where the channel tine is divided into contiguous slots of duration sufficiently long to transmit one packet of data [1]. If the packet length is variable then the slot length is also variable. This paper is concerned only with fixed packel size.

With base band signaling, two channels are required for the DSMA protocol, i.e., a main channel for data transmission and a subchannel to request data slot assignment. (If broadband signaling is employed, then data and slot request transmissions can be frequency multiplexed and transmitted over the same physical channel). Fach transceiver (TCVR) in the local area network is assigned an n-bit address a priori, and each has access to both of the channels as shown in Fig. 1.

To request a data slot allocation each active TCVR transmits its address bits serially with the most significant bit first into the common request channel. An active TCVR with an address lower than another aclive TCVR in the same request cycle drops off from contention temporarily until a slot has been assigned to the active TCVR with the highest address. Each TCVR is allowed access to only one data slot during a cycle; this ensures fairness for all TCVRs. A request cycle terminates when each active TCVR has been assigned a dala slot. Fig. 2 illustrates the slot request cycle.

A reservation period takes $h$ slots, which is the time required to transmit the n-bit address serially on the reservation channel. The end of a request cycle is indicated by imposing an $h$-slot silent period on the request channel [1]. Thus, if there are to be no gaps between consecutive DSMA cycles during a busy period, then the constraint $2 / 1<1$ must be satisfied [1]

Although the DSMA protocol is capable of multi-priority requests the analysis given in [1] has been concerned only with a single class message transmission without acknowledgement. A more complete model. which includes a prolocol for transmilling multi-priority messages with acknowledgement for each packet, is described in this paper. In addition, the possibility of integrating voice packet transmission is discussed. In the modified protocol the basic two channel configuration and the slot request rule of the original DSMA model remain essentially unattered.

Permission to copy without fee all or part of this material is granted provided that the copies are not made or distributed for direct commercial advantage, the ACM copyright notice and the title of the publication and its date appear, and notice is given that copying is by permission of the Association for Computing Machinery. To copy otherwise, or to republish, requires a fee and/or specific permission.

(c) 1983 ACM 0-89791-089-3/83/0300-0226 $\$ 00.75$
The paper is organized as follows. Section 2 explains the details of the modified protocol. Section 3 analyzes the delaythroughput characteristics. Analytical results are compared with simulation results in section 4 .

\section{ACKNOWLEDGING DSMA}

The frame format of DSMA is modified to permil the incorporation of fixed slot acknowledgement (FSA). The modified DSMA with fixed slot acknowledgement protocol will be referred to as DSMA/FSA.

\subsection{Acknowledgement Scheme}

The two channel timing diagram for the acknowledgemont scheme is shown in Fig. 3. A $b$-bil slot is reserved after every data slot for the transmission of an acknowledgement (ACK) packet. Takoro et al [2] have shown that it is not unreasonable for an ACK packet to be generated almost immediately upon reception of a data packet. (Acknowledging Ethernet is a modification of the Ethernet protocol [3], one that includes transmission of ACK packets for each packet.)

Acknowledgement for any given data packet should be Iransmitted $(1+a)$ slots $(a=b / P$, where $P$ is the length of one data packet in bits.) after the reception of a data packet. Since there is a fixed delay in the transmission of the ACK packet, it is apparent that every time an ACK packet is transmitted there is only one TCVR that has had the opportunity 10 create the packet, and there is only one TCVR in the entire network expecting acknowledgement. (If the source has multiple destinations, the $b$ bil acknowledgement mini slot may include a control field so that the source can designate which destination should acknowledge a given packet.) Thus, the size of the $b$-bit mini-slot can be made quite short; in fact, ideally only one bil ("yes" or "no") need be transmitted. All addressing information may be removed from the normal packet format. In practice, however, the "yes" or "no" signal consists of $b>1$ bits to enhance protection against channel noise, processing delay and other errors. For example, if $b=7$, we may use a $(7,4)$ Hamming code to protect the integrity of the ACK bits.

The fixed slor acknowledgement scheme (DSMA/FSA) possesses several advantages. Firstly, the length of the ACK packet can be made quite short relative to a data packet. Consequently, a very small proportion of the channel capacity is occupied by the ACK packets. Secondly, the format, and hence the generation of

* This work has been supported by the Natural Sciences and Engineering Research Council Canada under a Postgraduate Scholarship and Grant No. A7779

** J. W. Mark is now on sabbatical leave at Bell Laboratories, Murray Hill, New Jersey 07974. 
the ACK packets, is simple, consisting of a few ones or zeroes. Finally, the TCVR design is not complicated by the inclusion of high priority ACK packets that are of a different length and format from diata packets.

The one major disadvantage of the DSMA/FSA scheme is the wastage of data slots under certain conditions. For example, (see Fig. 3) if after the end of a cycle, a packet arrives during the period $B$, then it cannol be transmitted until after the following ACK slot (marked "A" in Fig. 3). Thus, the arrival of a data packet under this condition results in the wastage of a data slot and represents a penally in delay due to the DSMA/FSA scheme. A second more minor disadyantage of the DSMA/FSA scheme is that the first $b$-bit slot after an idle period is always wasted.

\section{2,2 Priority Sclieme}

Priority scheduling is accommodated in DSMA and DSMA/FSA by reserving the $k$ most significant bits of the $n$-bit TCVR address to indicale $K=2^{* *} k$ levels of priority [1]. The priority scheme will be described for lwo level $(k=1)$ priority. Extrapolation to $K$-level priority will then be straghtforward.

Any TCVR wishing to transmit a priority packel contends on the reservation bus by tratnsmilling its address bits, with the most significant bit of its n-bit address set to "1". This effectively makes the priority TCVR the highest address TCVR, hus ensuring transmission of the priority packet ahead of any non-priority packets with which it contends. If two or more TCVRs have priorily packet to be transmitted in the same cycle, the slot request rule applies within each level of priority. It should be noted that a priority packel arriving after the start of a scheduling interval must wait till the next scheduling interval before it can contend for access 10 the dita bus.

The operation of the two-level priority scheme described above is easily extended to $K$-level priority, where the highest priority TCVR would have all $k$-bits set to "l". As described above, the highest priority class packets would gain access to the data channel ahead of all lower priority class packets with which they contend.

\subsection{Voice Traffic Consideration}

Voice traffic characteristics are significantly diflerent from those of data traffic. Voice traffic consists of talkspurts and silent periods. During talkspurts, packels are generated periodically: during silences, no packet occur. The statistics of the duration of talkspurts and silences in conversational speech are given by Brady [4]. [5]. The mean talkspurt length and the mean pause length are $1.366 \mathrm{~s}$ and $1.802 \mathrm{~s}$, respectively. The call arrival distribution can be assumed to be Poisson [6], with the average duration of a call laken to be approximately 100s. Also, approximately $40 \%$ of the time a call is in talkspurt, with the talkspurt and silence lengths modeled using a geometric distribution [7].

There are two basic requirements for voice traffic: to maintain high quality speech the end-to-end network delays must be held to small fractions of a second and a nearly synchronous output must be maintained at the receiver output. Network delay is comprised of three components: terminal delays, nodal (TCVR) delays and propagation delay. Terminal and propagation delay will be ignored here and only the TCVR to TCVR protocol delay will be studied. (It should be noted, however, that in any real implementation, the terminal to TCVR protocol will incur some delay that will have to be considered.) It has been determined that a typical end-to-end delay of $150 \mathrm{~ms}-350 \mathrm{~ms}$ is satisfactory for delivering voice packets [8]. As well, gaps in conversations (interpacket delays) exceeding $50 \mathrm{~ms}$ should not occur for more than $1 \%$ of the packets [9].

To ensure that the criteria specified above are satisfied, the highest priority must be reserved for voice packets, i.e., voice packets will all be of higher priority than data packets. This means that amongst voice packets, the priority due to TCVR addresses will prevail. This inherent ordering of voice packets will have to be considered when determining whether the above criteria will be satisfied. (Actually, almost all systems, where channel access by users is position-dependent, are "unfair", unless a priorily swapping policy is incorporated.)

No acknowledgement is necessary for voice packets since comprehension of speech is quite tolerant of bit (packel) errors. It should, therefore, be possible to remove the $b$-bit acknowledgement mini slot described for the FSA scheme and utilize this bandwidth for voice packet transmission. This would, however, involve the adoption of a more complicated protocol fand hence more complex TCVR design), onc that would have to be able to adapt between interspersed data transmission (with acknowledgement) periods and voice transmission (without acknowledgment) periods. It appears that the advantage of a simpler TCVR and protocol may outweigh the marginal gain in bandwidth achievable by utilizing the $b$-bit acknowledgement slot ( $b$ in the order of 10 bits) for voice packet transmission. Voice packet transmission will, therefore, be integrated into the DSMA/FSA scheme where the acknowledgement slot corresponding to voice packets may or may not be used.

\section{DELAY ANALYSIS FOR DATA}

\subsection{DSMA/FSA Without Priority}

The analysis of the DSMA/FSA scheme follows closely the analysis of the DSM $A$ protocol [1]. Assumptions made in the derivation of an expression for packet delay of DSMA apply as well to the analysis below. Furthermore, all remarks and interpretations of the delay equation for DSMA hold for DSMA/FSA.

To obtain an expression for the packet delay, we consider a packet in the entire system and obtain a delay expression for any packet in the network. Following the arguments given in [1], the mean delay $\bar{d}$ may be expressed as:

$\vec{d}=$ propagation delay + service time

+ acknowledgement time + queueing delay.

The mean service time of a data packet cquals 1 stot and the acknowledgement time $=$ "a" slot. The mean queucing delay is given by the following:

\section{$\bar{W}=$ mean delay for packet to join the queue + average remaining service lime of packel in service + waiting time of $\bar{N}$ packets who are ahead of the packet in question.}

The mean delay for a packet to join the queue for service is given by:

$$
\begin{aligned}
\bar{H} & =h\left(1-\rho\left(1+\frac{a}{\bar{x}}\right)\right) e^{-\rho\left(1+\frac{a}{\bar{x}}\right)} \\
& +\left[1-\rho\left(1+\frac{a}{\bar{x}}\right)\right]\left[h+\frac{a+\bar{x}}{2}\right]\left[1-e^{-\rho\left(1+\frac{a}{\bar{x}}\right)}\right] .
\end{aligned}
$$

where $h$ in slots is the overhead due to reservation, $\bar{x}$ is the mean service time of a data packet, "a" in slots is the length of an ACK packet, and $\rho=\lambda \bar{x}$ is the traffic intensity and $\lambda$ is the mean aggregate arrival rate. The first term on the right-hand-side of (1) is the delay penalty when the system is idle and no packets arrive during the next $(1+a)$ slots. If at least one packet arrives during the next $(1+a)$ slots, a data slot is wasted and the delay penality is given by the second term on the right-hand-side of (1). Bearing in mind that the derivation of the delay equation follows the arguments given in $[1]$, without presenting details, the mean queueing delay $\bar{W}$ is given by

$\bar{W}=h+\left(\frac{1+a}{2}\right)\left(1-e^{-\rho^{\prime}}\right)+\frac{\rho^{\prime}(1+a)}{2\left(1-\rho^{\prime}\right)}$. 
where we have let $\bar{x}=1$ and $\rho^{\prime}=\rho(1+a)$. The mean packet delay $\bar{d}$ is now given by

$\bar{d}=\alpha+1+a+h+\left(\frac{1+a}{2}\right)\left(1-e^{-\rho}\right)+\frac{\rho^{\prime}(1+a)}{2\left(1-\rho^{\prime}\right)}$,

where $\alpha$ is the propagation delay in slots. Equation (3) only represents the delay to transmit a packet. The delay associated with reception of the acknowledgement packet has not been included since acknowledgement for each data packet arrives exactly $(1+a)$ slots after it has been transmitted, we may writc the mean delay as:

$\bar{d}=2 \alpha+2(1+a)+h+\left(\frac{1+a}{2}\right)\left(1-e^{-\rho^{\prime}}\right)+\frac{\rho^{\prime}(1+a)}{2\left(1-\rho^{\prime}\right)}$.

\subsection{DSMA/FSA with Priority}

The priority scheme implemented within DSMA/FSA may be modeled as a head-of-the-line (HOL) queueing system without preemption [11]. Thus, the derivation of an expression for a packet delay will follow the arguments outlined in 15, Chap. 3]. In what follows, we derive the delay expression for a two level priority system.

The mean queueing delay $\bar{W}$ may be expressed as:

$\bar{W}=\beta \bar{W}_{1}+(1+\beta) \bar{W}_{2}$

where

$\bar{W}_{1}=$ mean queueing delay for priority packets,

$\bar{W}_{2}=$ mean queueing delay for non-priority packets,

and

$\beta=$ fraction of total traffic that is priority.

The traffic into a two-level priority HOL system may be taken as two independent Poisson streams with intensity $\rho_{1}^{\prime}=\rho_{1}(1+a)$ and $\rho^{\prime}{ }_{2}=\rho_{2}(1+a)$ such that $\rho^{\prime}=\rho_{1}^{\prime}+\rho_{2}^{\prime}$, where $\rho_{1}^{\prime}=$ traffic intensity of priority packets and $\rho_{2}^{\prime}=$ traffic intensity of non-priority packets. The same result may be achieved by considering the input traffic stream, with intensity $\rho^{\prime}$, where a fraction $\beta$ of the packets are randomly and independently lagged to be priority and the remainder are tagged to be non-priority packets. Therefore, we get $\beta=\rho_{1}^{\prime} / \rho^{\prime}$ and $(1-\beta)=\rho^{\prime} / \rho^{\prime}$ and equation (5) may be written as:

$\bar{W}=\frac{\rho_{1}^{\prime}}{\rho^{\prime}} \bar{W}_{1}+\frac{\rho_{2}^{\prime}}{\rho^{\prime}} \bar{W}_{2}$

Following the arguments in the derivation of the expression for $\bar{W}$ in the single class commodity case, we obtain

$$
\begin{aligned}
\bar{W}_{1}= & {\left[\frac{1-\rho^{\prime}}{1-\rho_{1}^{\prime}}\right]\left[h+\left(\frac{1+a}{2}\right)\left(1-e^{\left.-\rho^{\prime}\right)}\right]\right.} \\
& +\frac{\rho^{\prime}(1+a)}{2\left(1-\rho_{1}^{\prime}\right)}+(1+a)\left[1-\left(1-\rho^{\prime}\right) e^{-\rho^{\prime}}\right] \rho_{2}^{\prime} .
\end{aligned}
$$

Now, using the relationship for $W$ given by (6), we may solve for $\bar{W}$ to obtain:

$$
\begin{aligned}
\bar{W}_{2}= & {\left[h+\left(\frac{1+a}{2}\right)\left(1-e^{-\rho^{\prime}}\right)+\frac{\rho^{\prime}(1+a)}{2\left(1-\rho^{\prime}\right)}\right]\left[\frac{1}{1-\rho_{1}^{\prime}}\right] } \\
& -(1+a)\left[1-\left(1-\rho^{\prime}\right) e^{-\rho}\right] \rho_{1}^{\prime},
\end{aligned}
$$

where

$\vec{W}=h+\left(\frac{1+a}{2}\right)\left(1-e^{-\rho}\right)+\frac{\rho^{\prime}}{2\left(1-\rho^{\prime}\right)}$.

Finally, the equations for packet delay may be obtained from (4) by substituting the appropriate expression for mean queueing delay. The mean delay for the transmission of a priority packet is given by

$$
\begin{aligned}
\bar{d}_{1}= & \alpha+1+a+\left[\frac{1-\rho^{\prime}}{1-\rho^{\prime}}\right]\left[1+\left(\frac{1+a}{2}\right)\left(1-e^{-\rho^{\prime}}\right)\right] \\
& +\frac{\rho^{\prime}(1+a)}{2\left(1-\rho_{1}\right)}+(1+a)\left[1-\left(1-\rho^{\prime}\right) e^{-\rho^{\prime}}\right] \rho^{\prime}{ }_{2}
\end{aligned}
$$

and that for a non-priority packet is given by

$$
\begin{aligned}
\bar{d}_{2}= & \alpha+1+a+\left[h+\left(\frac{1+a}{2}\right)\left(1-e^{-\rho}\right)+\frac{\rho^{\prime}(1+a)}{2\left(1-\rho^{\prime}\right)}\right]\left[\frac{1}{1-\rho^{\prime}{ }_{1}}\right] \\
& -(1+a)\left[1-\left(1-\rho^{\prime}\right) e^{-\rho^{\prime}}\right] \rho_{1}^{\prime} .
\end{aligned}
$$

When the delay for reception of acknowledgement is taken into account, equations (9) and (10) become, respectively,

$$
\begin{aligned}
\bar{d}_{1}= & 2(\alpha+1+a)+\left[\frac{1-\rho^{\prime}}{1-\rho_{1}^{\prime}}\right]\left[1+\left(\frac{1+a}{2}\right)\left(1-e^{-p^{\prime}}\right)\right] \\
& +\frac{\rho^{\prime}(1+a)}{2\left(1-\rho_{1}\right)}+(1+a)\left[1-\left(1-\rho^{\prime}\right) e^{-\rho^{\prime}}\right] \rho_{2}^{\prime}
\end{aligned}
$$

and

$$
\begin{aligned}
\bar{d}_{2}= & 2(\alpha+1+a)+\left[h+\left(\frac{1+a}{2}\right)\left(1-e^{-\rho^{\prime}}\right)+\frac{\rho^{\prime}(1+a)}{2\left(1-\rho^{\prime}\right)}\right]\left[\frac{1}{1-\rho_{1}^{\prime}}\right] \\
& -(1+a)\left[1-\left(1-\rho^{\prime}\right) e^{-\rho^{\prime}}\right] \rho_{1}^{\prime} .
\end{aligned}
$$

\section{NUMERICAL RESULTS}

Voice is synchronous traffic. The delay-throughput performance for data can be studied under a reduced channel capacity model, where the capacity reduction is the portion of the total capacity captured by voice, plus any overhead due to boundary switching between voice and data transmissions. The numerical results presented in this section is restricted to Poisson input traffic only. 
Analytical and simulation results of delay-throughpul performance for DSMA/FSA are plotled in Fig. 4. The results were obtained for a slot length equal to the length of a 256 bit data packet and an ACK packel length of $b=10$ bils. Transceiver address length of $n=8$ bits, giving a request overhead of $\mathrm{l}=$ 0.3164 slot, was used. Using the same parameters, analyticat and simulation results for a two level priority system are plotted in Fig. 5.

The analytical results are in close agreement with the simulation results. Only for the case of $85^{1 /} \%$ priority traffic is there any disagreement between simulation and analysis. Even in this case, there is still "good" agreement belween simulation and analysis at low to medium throughpul ranges, i.c. at throughout rates $<0.7$

Suppose the priority commodity is voice and the transmission rate is 2 Mbps. Consider the case that $50 \%$ of the total traffic is priorily traffic. Al $\rho=0.80$, we obtain from Fig. 5 that the mean packet delay for the priority (voice) traffic is approximately 3.5 slots, which corresponds to approximately $0.5 \mathrm{~ms}$.

\section{CONCLUSIONS}

In this paper we have described a fixed slot acknowledgement scheme for the distributed scheduling mulliple access (DSMA) protocol described in [1]. Although there is a wastage of data slot when packets arrive within one slot after the system becomes idle. the proposed DSMA/FSA scheme is more efficient in terms of delay-throughput compared to the case in which a separate acknowledgement packet is sent for each data packet.

Since DSMA offers multi-priority requests, the TCVRs in the local area network are coordinated so that priority scheduling can be imposed at the communications subnetwork level, j.e.. not just at an individual TCVR. With integrated voice and data services, voice Iraffic can be treated as a high priority network commodity. It is conjectured that DSMA/FSA is capable of supporting integrated voice-data services which meet the end-to-end mean packel delay and mean interpacket delay requirements for voice.

\section{REFERENCES}

(1) J. W. Mark, "Distributed Scheduling Conflict-Free Multiple Access for Local Area Communication Networks," IEEE Trans. Comm. Vol. COM-28, No. 12, pp. 1968-1976. Dec. 1980 .

[2] M. Takoro, el al, "Acknowledging Elhernet," Compcon, Fall'77, pp. 320-325.

[3] D. Boggs and R. Metchalfe, "Ethernet: Distributed Packel Swilching for Local Computer Networks," Comm. $A C M$, Vol. 19. pp. 395-404, July 1976.

[4] P. T. Brady, "A Technique for Investigating On-Of Palterns in Speech," BSTJ, Vol. 44, pp. 1-2, 1963.

[5] .........."Effects of Transmission Delay on Conversational Behavior on Echo-Free Telephone Circuits," BSTJ, Vol. 50. pp. 115-134, 1971

[6] S. Q. Li, "Performance Analysis of a Conflict-Free Multiple Access Local Area Network for Voice and Data". M.A.Sc. thesis. Dept. of Elecurical Engineering, Univ, of Waterloo, Waterloo, Ontario, Canada, 1981.

[7] N. Janakirman, B. Pagurek and J. E. Neilson, "Multiplexing Voice Sources," IEEE NTC'81 Vol. 4, pp. G4.3.1-G4.3.5

[8] G. J. Coviello, O. L. Lake and G. R. Redinbo. "System Design Implications of Packetized Voice", Proc. ICC'77, pp. 38.3-1 to 38.3-5, June 1977, Chicago, ll.

[9] E. Gopal, J. W. Wong and J. C. Majithia. "An Evaluation of Playout Schemes for Voice Transmission in Packet Networks," Computer Nelworking Symposium. Gaithersburg, Maryland, Dec. 1981.

[10] L. Kleinrock, Queucing Systems: Volume I, John Wiley \& Sons, New York, 1975

[11] -...-.... Queneing Strstems: Volume II, John Wilcy \& Sons, New York, 1976

[12] J. D. C. Litle, "A Proof of the Queucing Formulit L $=w$," Operations Research, Vol. 9, pp. 383-387, 1961.

HIGH SPEED DATA BUS

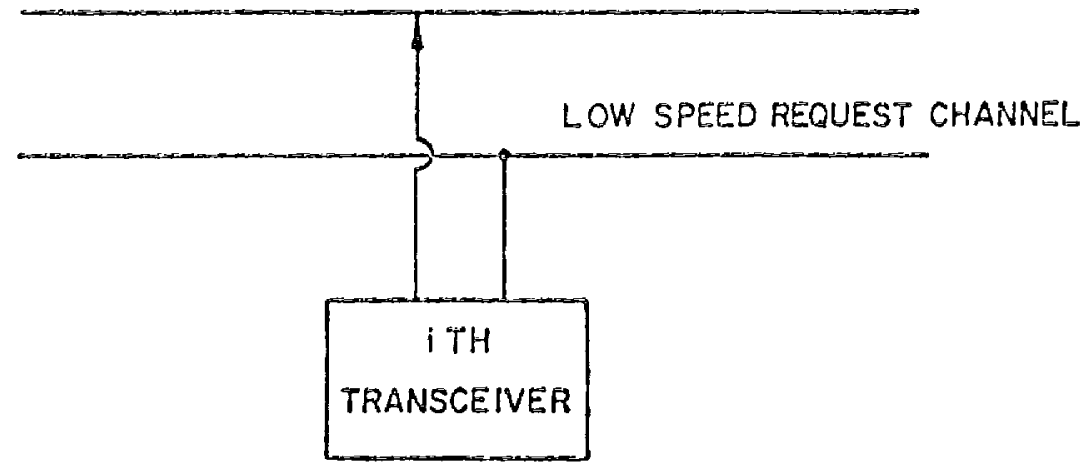

Figure 1 Dual Channel Access by iTH Transceiver 


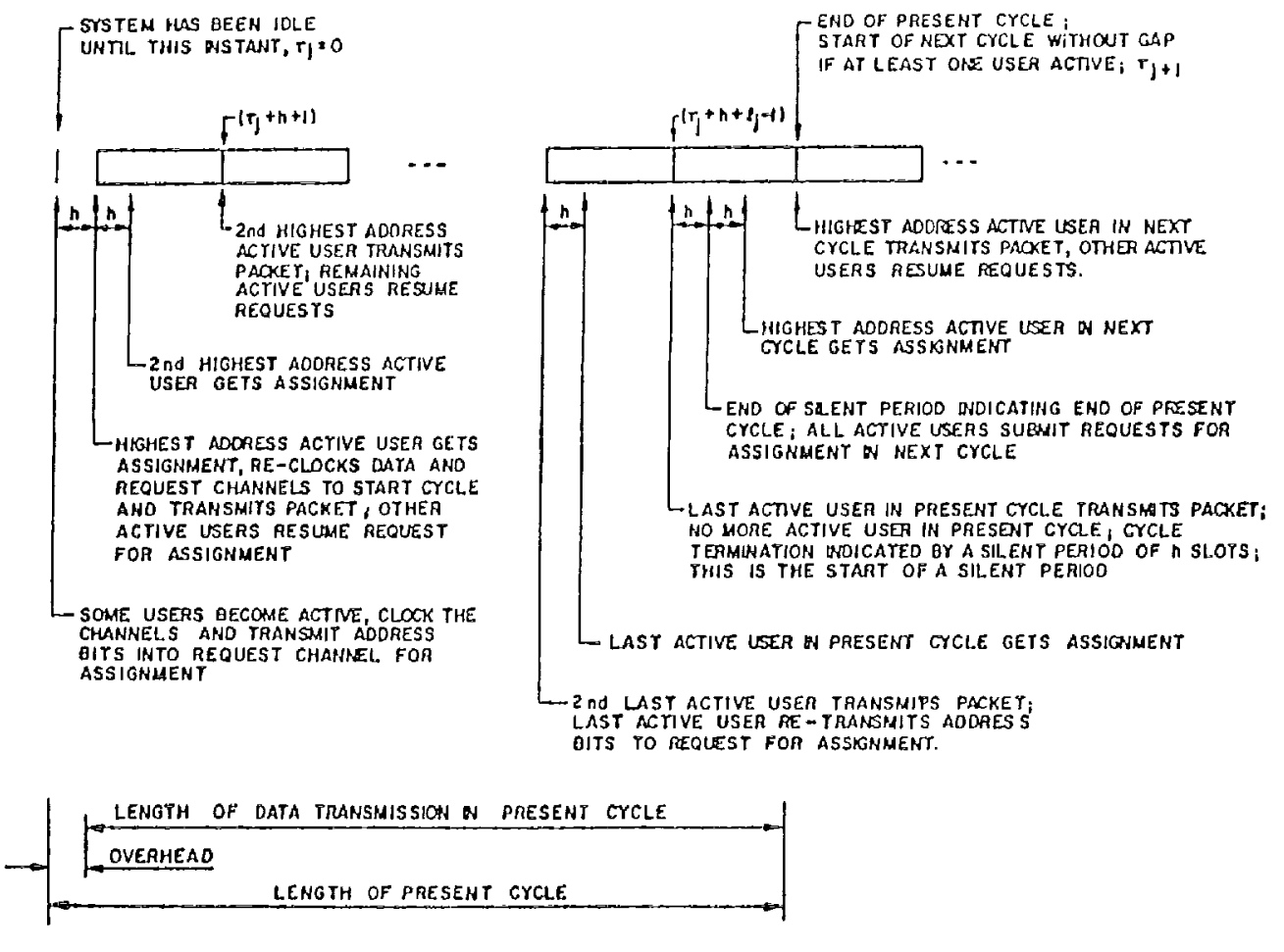

Figure 2 Slot Request Rule

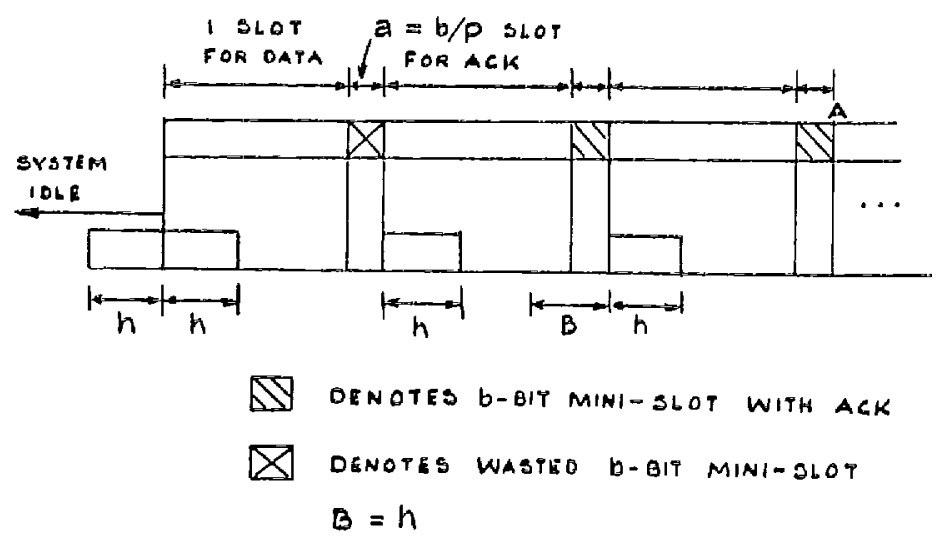

Figure 3 DSMA/FSA TIming Diagram 


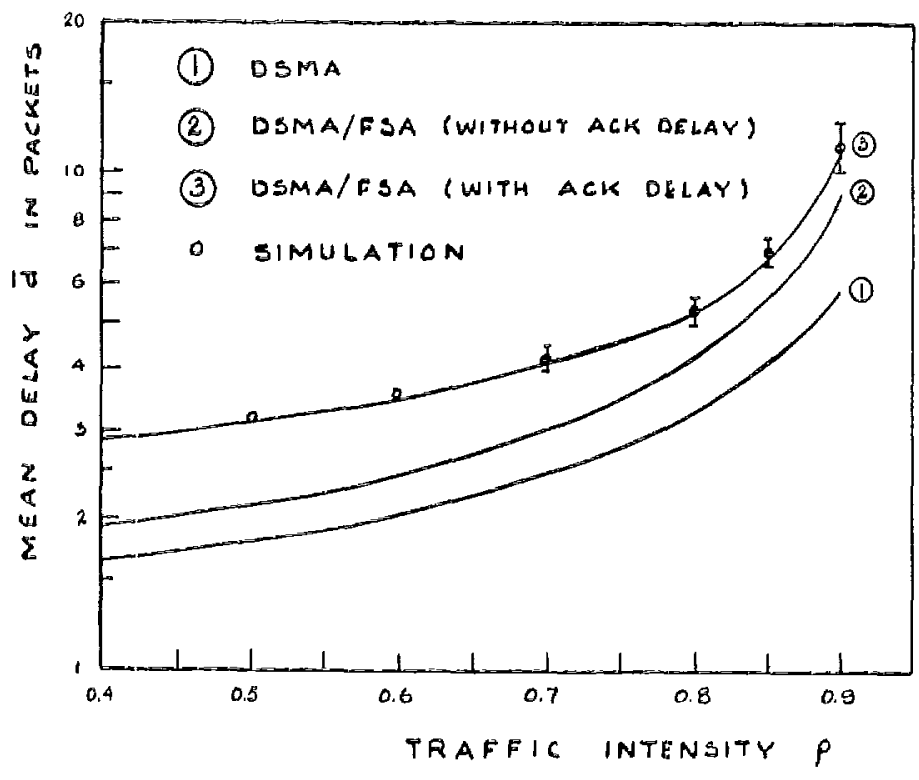

Figure 4 DSMA/FSA Delay-Throughput

characteriatics

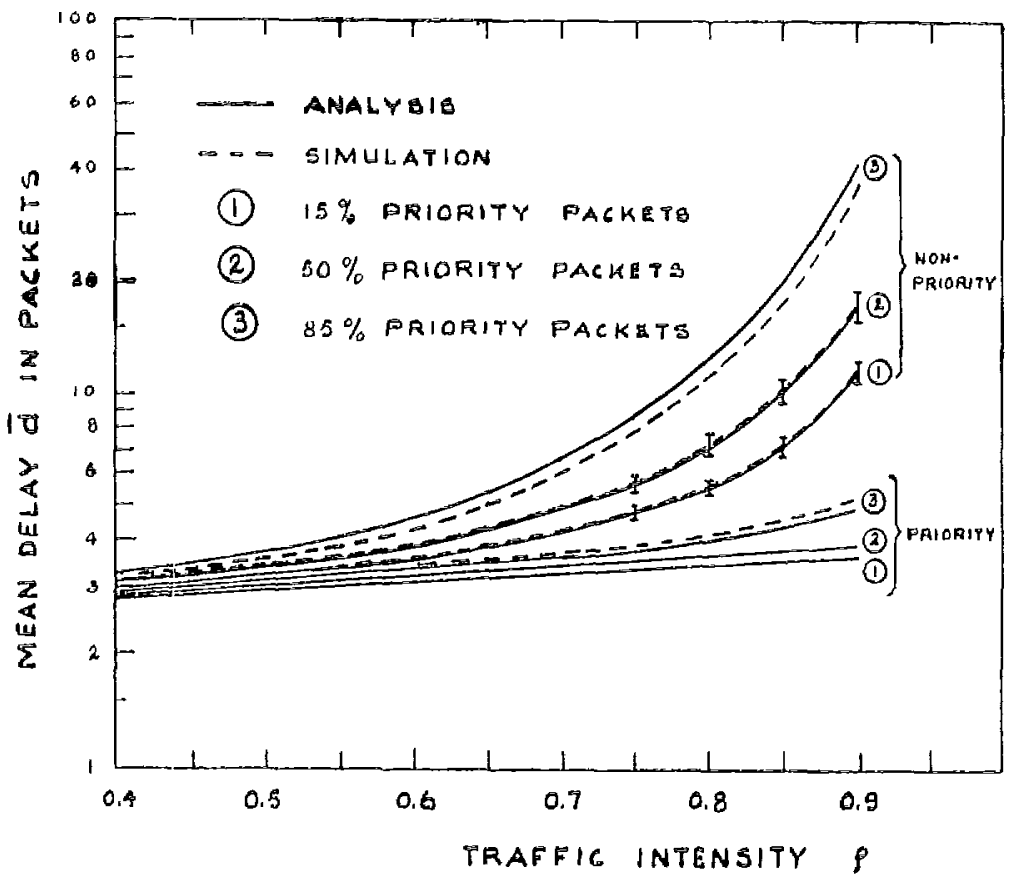

Figures Delay-ihroughput characterloticos of OSMA/FSA with Priority 